\title{
NOTCH pathway inactivation promotes bladder cancer progression
}

\author{
Antonio Maraver, ${ }^{1}$ Pablo J. Fernandez-Marcos, ${ }^{1}$ Timothy P. Cash, ${ }^{1}$ Marinela Mendez-Pertuz, ${ }^{2}$ Marta Dueñas, ${ }^{3}$ Paolo Maietta, ${ }^{4}$ \\ Paola Martinelli, ${ }^{2}$ Maribel Muñoz-Martin, ${ }^{1}$ Mónica Martínez-Fernández, ${ }^{3}$ Marta Cañamero, ${ }^{5}$ Giovanna Roncador, ${ }^{6}$ \\ Jorge L. Martinez-Torrecuadrada, ${ }^{7}$ Dimitrios Grivas, ${ }^{8}$ Jose Luis de la Pompa, ${ }^{8}$ Alfonso Valencia, ${ }^{4}$ Jesús M. Paramio, ${ }^{3}$ \\ Francisco X. Real, ${ }^{2,9}$ and Manuel Serrano ${ }^{1}$ \\ 'Tumor Suppression Group and 'Epithelial Carcinogenesis Group, Spanish National Cancer Research Centre (CNIO), Madrid, Spain. ${ }^{3}$ Molecular Oncology Unit, Centro de Investigaciones Energéticas, \\ Medioambientales y Tecnológicas (CIEMAT), and Oncogenomic Unit, University Hospital “12 de Octubre," Madrid, Spain. "Structural Computational Biology Group, ${ }^{5}$ Comparative Pathology Unit, \\ ${ }^{6}$ Monoclonal Antibody Unit, and ${ }^{7}$ Proteomics Unit, CNIO, Madrid, Spain. ${ }^{8}$ Intercellular Signaling in Cardiovascular Development and Disease Group, Centro Nacional de Investigaciones Cardiovasculares, \\ Madrid, Spain. ${ }^{9}$ Universitat Pompeu Fabra, Barcelona, Spain.
}

\begin{abstract}
NOTCH signaling suppresses tumor growth and proliferation in several types of stratified epithelia. Here, we show that missense mutations in NOTCH1 and NOTCH2 found in human bladder cancers result in loss of function. In murine models, genetic ablation of the NOTCH pathway accelerated bladder tumorigenesis and promoted the formation of squamous cell carcinomas, with areas of mesenchymal features. Using bladder cancer cells, we determined that the NOTCH pathway stabilizes the epithelial phenotype through its effector HES1 and, consequently, loss of NOTCH activity favors the process of epithelial-mesenchymal transition. Evaluation of human bladder cancer samples revealed that tumors with low levels of HES1 present mesenchymal features and are more aggressive. Together, our results indicate that NOTCH serves as a tumor suppressor in the bladder and that loss of this pathway promotes mesenchymal and invasive features.
\end{abstract}

\section{Introduction}

NOTCH is a single-pass transmembrane receptor activated by interaction with transmembrane ligands of the DSL (Delta-like and Jagged) family present on the membrane of neighboring cells. There are 4 different NOTCH receptors (NOTCH1, $-2,-3$, and -4) and 5 ligands (JAGGED1 and -2 and DLL1, -3 , and -4 ) in humans. Ligand binding to the extracellular domain of NOTCH induces a cascade of proteolytic cleavages ending with the processing by the $\gamma$-secretase complex and the release of the NOTCH intracellular domain. The NOTCH intracellular domain translocates to the nucleus, where it binds the transcription factor RBPJ, and the resulting complex activates the transcription of target genes, notably including transcriptional repressors of the HES and HEY family (1).

Alterations of the NOTCH pathway are frequent in multiple cancers. Paradoxically, these alterations can be grouped into two patterns with opposite functional effects, and each pattern is associated with specific tumor types (2). In particular, gain-offunction mutations of the NOTCH pathway are present in acute T cell lymphoblastic leukemia, chronic lymphocytic leukemia, and lung adenocarcinoma, indicating that the NOTCH pathway is oncogenic in these malignancies (3-5). On the contrary, loss-of-function mutations are observed in myeloid leukemia and in squamous cell carcinomas (SCCs) of different origins (head

Authorship note: Antonio Maraver and Pablo J. Fernandez-Marcos contributed equally to this work.

Conflict of interest: The authors have declared that no conflict of interest exists. Submitted: July 23, 2014; Accepted: December 4, 2014.

Reference information: / Clin Invest. 2015;125(2):824-830. doi:10.1172/JCI78185. and neck, lung, and skin), thereby implying that the NOTCH pathway plays a tumor-suppressive role in these cancer types (6-9). However, how the NOTCH pathway protects from squamous cancers is still incompletely understood.

Bladder cancer is a major health burden worldwide (10). The majority of bladder tumors arise in the urothelium, a specialized stratified epithelium, and a fraction of bladder cancers display squamous features, corresponding to a more aggressive form of bladder cancer (11). Taking into account that the NOTCH pathway is tumor suppressive in several types of SCCs, we hypothesized that this could also be the case in the urothelium.

Here, we demonstrate that patients with bladder cancer harbor loss-of-function mutations in both NOTCH1 and NOTCH2 receptors. In addition, genetic inactivation of the NOTCH pathway in the urinary bladders of mice by two different genetic means accelerates bladder cancer and promotes the formation of SCCs. Mechanistically, we show that loss of the NOTCH pathway promotes an epithelial-mesenchymal transition (EMT) in bladder cancer cells and this is partly mediated by loss of HES1. Finally, human bladder cancers with low levels of HES1 present mesenchymal features and have an aggressive phenotype.

\section{Results}

Loss-of-function mutations of NOTCH1 and NOTCH2 in bladder cancer. Genome-wide analyses of bladder cancer by others and us have identified mutations in NOTCH receptors (12-14); however, their functional meaning has remained unexplored. Missense mutations in the extracellular EGF repeats of NOTCH1 and NOTCH2 have been reported in skin, lung, and head-and-neck 
A
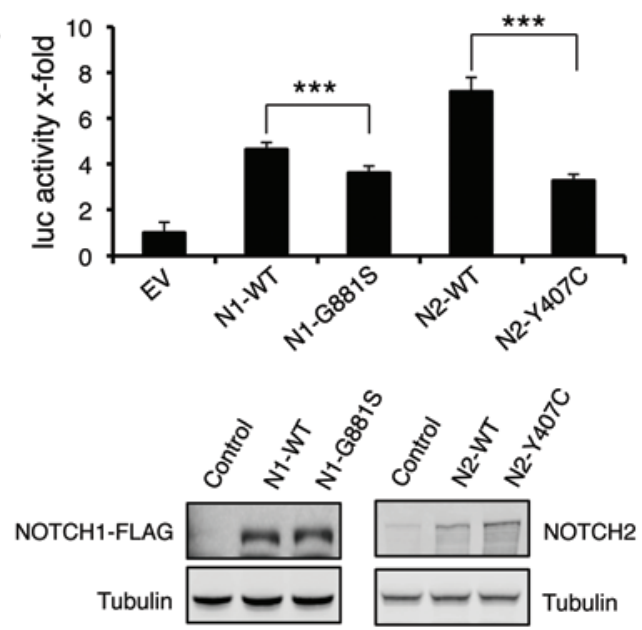

B

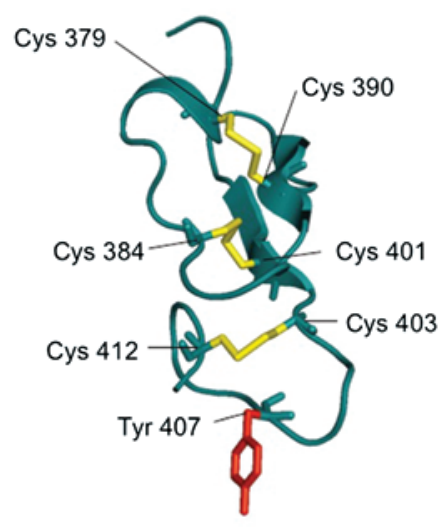

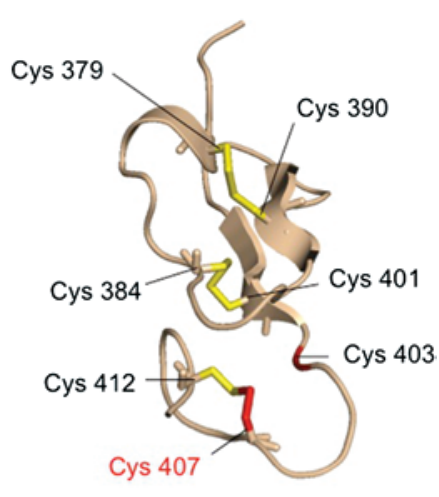

Figure 1. NOTCH1 and NOTCH2 missense mutations found in human bladder carcinomas lead to decreased NOTCH activity. (A) T24 cells were cotransfected with a plasmid carrying a luciferase reporter gene directed by an RBPJ-responding promoter and with constructs carrying the indicated versions of NOTCH1 (N1) and NOTCH2 (N2). Subsequently, they were exposed to HEK293T cells overexpressing the NOTCH ligand JAGCED1 to activate NOTCH signaling and, consequently, luciferase expression. Bars represent the average of 5 independent measurements of luciferase, and error bars represent SD. The levels of overexpression were assessed by immunoblot (tubulin and FLAG immunoblots from the left panel were performed in two different but equivalent membranes; see complete unedited blots in the supplemental material). ${ }^{* *} P<0.001$, Student's $t$ test. EV, empty vector. (B) Predicted structural effect of the NOTCH2-Y407C mutation is marked in red. Disulfide bridges predicted in WT and in NOTCH2-Y407C are marked in yellow.

SCCs (refs. 6-8 and Supplemental Figure 1; supplemental material available online with this article; doi:10.1172/JCI78185DS1). To examine the functional effect of human bladder cancer-associated mutations in NOTCH1 and NOTCH2, we chose the 2 mutations with the highest damaging prediction among those found by us in a previous exome-sequencing project (13), namely, NOTCH1-G881S and NOTCH2-Y407C (Supplemental Figure 1). The 2 mutant NOTCH1 and NOTCH2 cDNAs were overexpressed at similar levels in 2 different cell lines, T24 bladder cancer cells and HEK293T cells, and both triggered significantly reduced activity of an RBPJ-dependent luciferase reporter in comparison with their corresponding WT cDNAs (Figure 1A and Supplemental Figure 2). Interestingly, in silico modeling of the NOTCH2-Y407C mutation predicted a change in the pattern of disulfide bridges in a region very close to the ligand interaction domain (ref. 15 and Figure 1B), and this could be the structural basis of its decreased activity. Together, these findings suggest that the NOTCH pathway plays a tumor-suppressive role in bladder cancer.

NOTCH pathway inactivation promotes bladder cancer in mice. To analyze the role of the NOTCH pathway in bladder carcinomas, we inactivated the pathway in the bladders of mice using two different strategies. First, we inactivated the two Psen paralogs, which encode the presenilin proteases essential for the activation of all the NOTCH receptors (16). In a complementary approach, we eliminated the Rbpj gene encoding the transcriptional factor critical for the canonical effects of the NOTCH pathway (17). More specifically,
Figure 2. NOTCH inactivation promotes bladder cancer in mice. (A) Kaplan-Meier survival curves representing the life span of WT $(n=8)$, PsenKO $(n=8)$, and RbpjKO $(n=10)$ mice inoculated intravesically with adeno-Cre and, subsequently, given $\mathrm{N}$-butyl- $\mathrm{N}$ (4-hydroxybutyl)nitrosamine in the drinking water for 20 weeks. ${ }^{* *} P<0.01$, log-rank test. (B) Bladder samples from WT $(n=5)$, PsenKO ( $n=7)$, and RbpjKO ( $n=7)$ mice were examined, and their lesions were graded. ${ }^{*} P<0.05$, Student's $t$ test. (C) Samples from the same mice as in $\mathbf{B}$ were histologically analyzed to determine the presence of SCC features. ${ }^{*} P<0.05$, Fisher's exact test. (D) Immunohistochemical stainings of the same bladder samples as in B and $\mathbf{C}$ were scored for the expression of the indicated proteins. ${ }^{*} P<0.05,{ }^{* *} P<0.01,{ }^{* *} P<0.001$, Student's $t$ test.
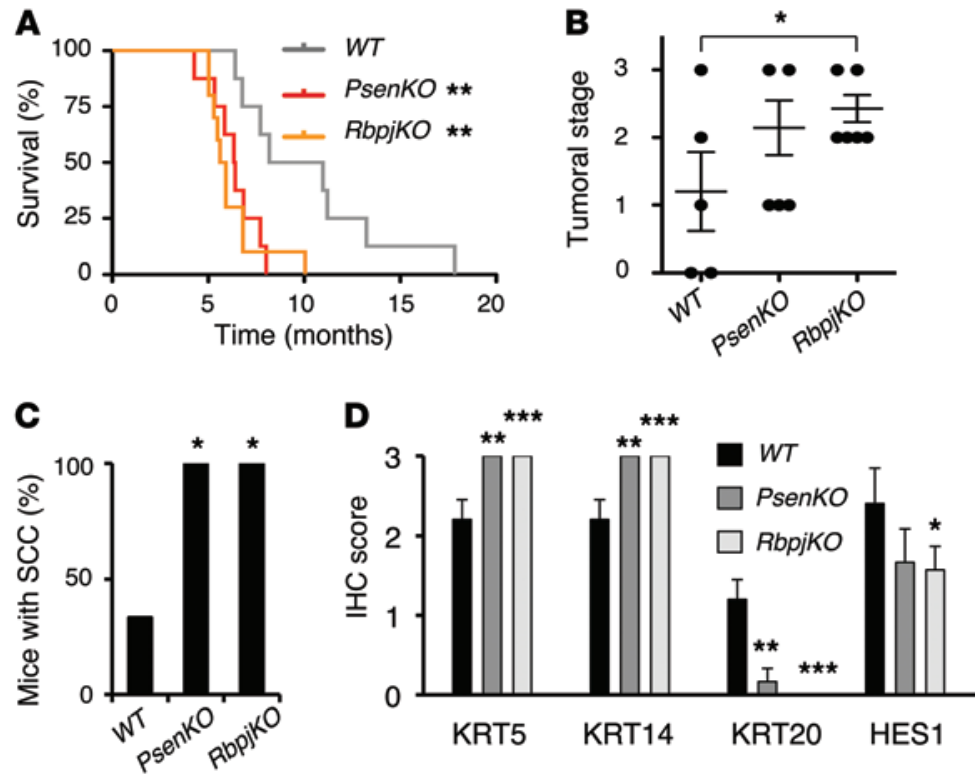
A

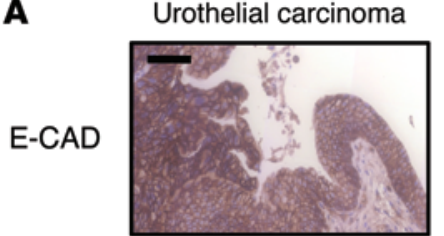

VIM

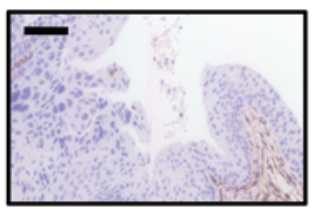

HES 1

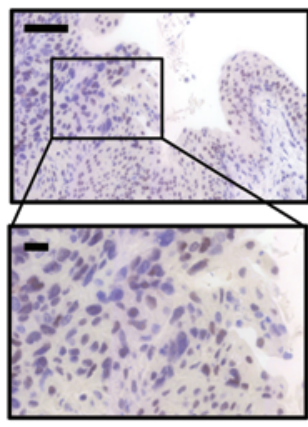

Mesenchymal region
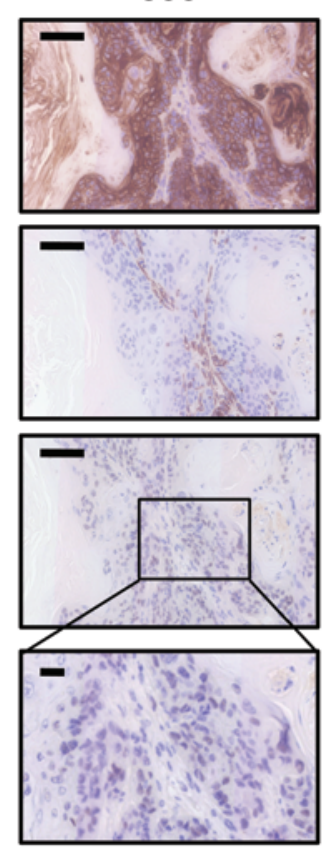
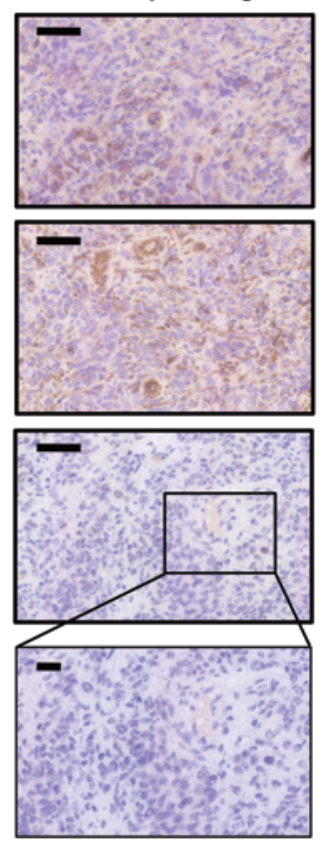

B

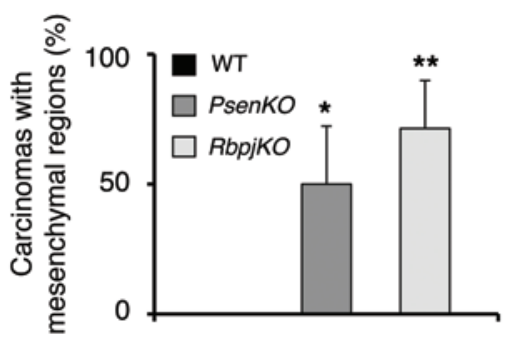

we used mice carrying a Cre-excisable allele of Psen 1 and null for Psen 2 (Psen $1^{A / f} P s e n 2^{-/-}$mice; referred to herein as PsenKO mice) (18) or mice carrying a Cre-excisable allele of $R b p j\left(R b p j^{p / f l}\right.$ mice; referred to herein as RbpjKO mice) (19). These mice, together with their corresponding WT littermates, were intravesically injected with adeno-Cre to induce the deletion of the excisable genes specifically in the bladder urothelium (20). To assess the efficiency of the Cre recombinase in the urothelium, we used Rosa26::LSL-LacZ mice carrying a silent $L a c Z$ reporter that becomes active upon Cremediated excision (21). As expected, PBS-treated Rosa26::LSL-LacZ mice were completely negative upon staining for LacZ (Supplemental Figure 3). In contrast, adeno-Cre-treated Rosa26::LSL-LacZ mice presented patches of the urothelium with intense LacZ staining that reached the urothelial basal layer, which is of relevance because bladder cancer-initiating cells reside in the basal layer of the bladder (ref. 22 and Supplemental Figure 3).

To evaluate the impact of NOTCH pathway inactivation on bladder cancer, PsenKO, RbpjKO, and WT mice were exposed for 20 weeks to the carcinogen N-butyl-N-(4-hydroxybutyl)nitrosamine, which is a commonly used experimental carcinogen with high selectivity for the urothelium $(23,24)$. As expected, all mice, regardless of their genotype, developed bladder tumors, and we only found tumors of urothelial origin. Interestingly, inactivation of the NOTCH pathway in PsenKO or RbpjKO mice resulted in a significant acceleration of blad-

Figure 3. NOTCH inactivation induces the appearance of mesenchymal regions in SCCs. (A) Representative examples of IHC stainings against the indicated proteins on the indicated tumor subtypes and regions. Note the drastic decrease of HES1 and E-cadherin and the increase of vimentin in the mesenchymal region. Scale bars: $50 \mu \mathrm{m}$ (top 3 rows); $20 \mu \mathrm{m}$ (bottom row). (B) Quantification of the percentage of bladder carcinomas (WT, $n=5$; PsenKO, $n=7 ; \operatorname{RbpjKO}, n=7$ ) presenting a mesenchymal region in mice of the indicated genotypes, assessed by the loss of E-cadherin. ${ }^{*} P<0.05,{ }^{* *} P<0.01$, Student's $t$ test.

der tumorigenesis and, thereby, shorter survival (Figure 2A). Tumors were classified according to current standard histological criteria (10), which include noninvasive tumors (stage 0 ), invasion of the underlying lamina propria (stage 1), invasion of the muscular layer (stage 2), and involvement of perivesical tissues (stage 3) (see examples in Supplemental Figure 4). Importantly, the inactivation of the NOTCH pathway was associated with the development of more invasive tumors (stages 2 and 3) (Figure 2B). This was further substantiated by the observation of bladder cancer infiltration into the prostates of PsenKO mice (2 of 4) and $R b p j K O$ mice (2 of 5), which in some cases was perineural (Supplemental Figure 5). In contrast, none of the WT mice (0 of 4) had cancer infiltration into the prostate. The urothelial origin of the cancer cells infiltrating the prostate was confirmed by their positive staining for KRT14 (Supplemental Figure 5), which is a bona fide marker of stratified epithelia (25). Moreover, we confirmed that intravesical adeno-Cre does not reach the prostate, as indicated by the absence of LacZ staining in the prostates of adeno-Cre-infected Rosa26::LSL-LacZ mice (Supplemental Figure 6).

Histologically, the PsenKO and RbpjKO tumors were diagnosed as SCCs (100\%) (Figure 2C and Supplemental Figure 7). In contrast, in the case of WT mice, a fraction of tumors (33\%) were SCCs and the rest were urothelial carcinomas (Figure $2 \mathrm{C}$ and Supplemental Figure 7). Supporting the above classification, overall quantification of immunohistochemical stainings indicated that, compared with WT tumors, PsenKO and RbpjKO tumors displayed a typical pattern of SCC markers. In particular, they were high in keratins KRT5 and KRT14 and negative for keratin KRT20 (Figure 2D and Supplemental Figure 8). In addition, as expected, the overall levels of HES1 were reduced in $P s e n K O$ and $R b p j K O$ tumors (Figure 2D).

We conclude that genetic inactivation of the NOTCH pathway in mice accelerates bladder carcinogenesis and favors the formation of highly invasive SCCs.

Tumors with deficient NOTCH pathway display EMT features. Interestingly, all the PsenKO and RbpjKO SCCs presented regions of mesenchymal morphology that lacked E-cadherin and expressed vimentin (Figure 3A). These mesenchymal regions coexisted together with the predominant SCC component of PsenKO and RbpjKO tumors, characterized by high levels of E-cadherin and absence of vimentin (Figure 3A). Importantly, regions of mesenchy- 


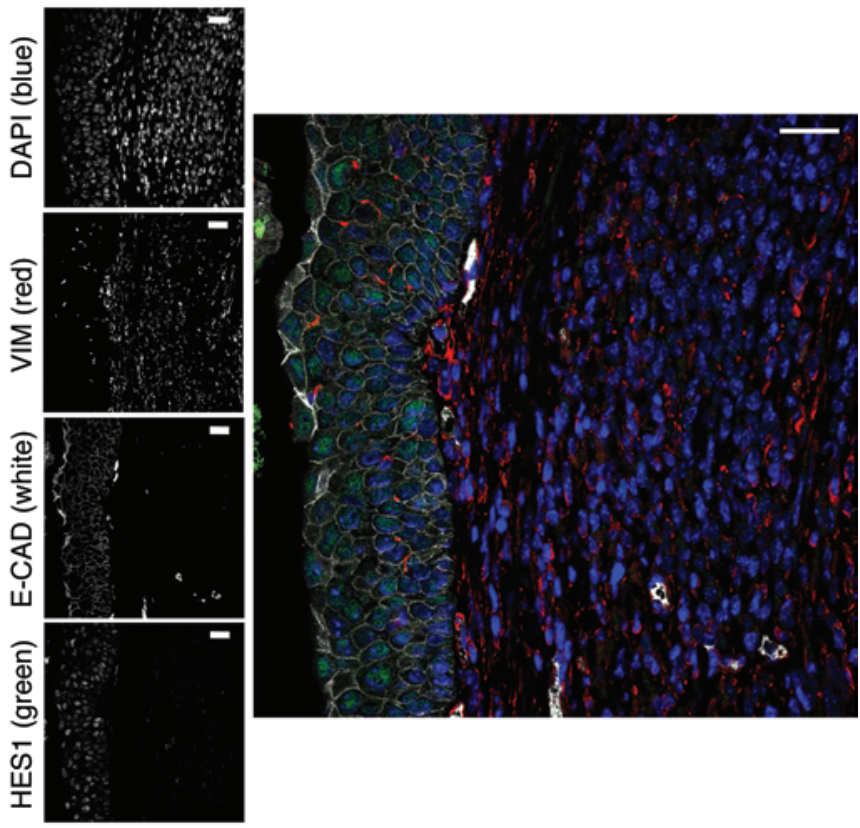

Figure 4. HES1 and E-cadherin colocalize in well-differentiated urothelium and are lost in vimentin-expressing mesenchymal regions.

Immunofluorescence with the indicated antibodies was performed on a mesenchymal region from a PsenKO mouse, with adjacent remnants of well-differentiated urothelium (left) as an internal control for HES1 and E-cadherin expression. Scale bars: $25 \mu \mathrm{m}$.

mal morphology were only found in PsenKO and RbpjKO tumors but not in WT tumors (Figure 3B). We, along with others, have implicated the transcriptional repressor HES1 as a relevant mediator of the NOTCH pathway in cancer (26-29). Remarkably, HES1 was clearly detected in the urothelial carcinomas present in WT mice, it was notably decreased in the SCCs, and it was essentially absent in the mesenchymal regions of the SCCs (Figure 3A). To further support the relationship between HES1 and EMT, we performed immunofluorescence on paraffin sections of murine bladder carcinomas presenting a mesenchymal component. Interestingly, we observed that HES1 colocalizes with E-cadherin in the adjacent normal urothelium, but both were absent in the mesenchymal areas expressing vimentin (Figure 4). Together, these observations suggest that NOTCH pathway inactivation favors EMT in bladder SCCs.

To extend the above findings to human cancers, we performed data mining of the exome sequencing and RNA sequencing data for human bladder carcinomas available at The Cancer Genome Atlas (TCGA) (12). Our analyses indicated NOTCH1 and NOTCH2 mutant cancers clustered in a group defined by squamous molecular features (Fisher's exact test, $P=0.02$ ) and showed a tendency to have increased levels of the squamous markers KRT5, KRT14, and TP63 (Supplemental Figure 9A). Of note, NOTCH1 and $\mathrm{NOTCH} 2$ mutant cancers showed a tendency toward a decrease in NOTCH targets (HES1 and HEY1, Supplemental Figure 9B), which is in agreement with our previous functional characterization of NOTCH1 and NOTCH2 mutants (Figure 1 and Supplemental Figure 2). Interestingly, NOTCH1 and NOTCH2 mutant cancers expressed markedly lower levels of E-cadherin (CDH1) and higher levels of vimentin (VIM) (Supplemental Figure 9C). Analysis of the allele frequency for these missense mutations revealed that they were heterozygous (allele frequency lower than 0.5 , with at least $60 \%$ of tumoral cells in the sample). This implies that just one mutant allele of NOTCH1 or NOTCH2 (with one WT allele still present) is sufficient to promote SCC bladder cancer. Collectively, these observations indicate that reduced NOTCH signaling favors squamous histology and the acquisition of mesenchymal features in murine and human bladder cancers.

NOTCH inactivation promotes EMT in a cell-autonomous manner. To explore the mechanism behind the association between NOTCH pathway inactivation and the acquisition of mesenchymal cellular
A

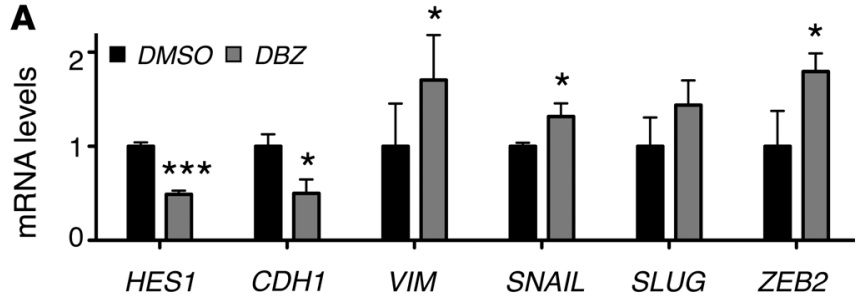

B

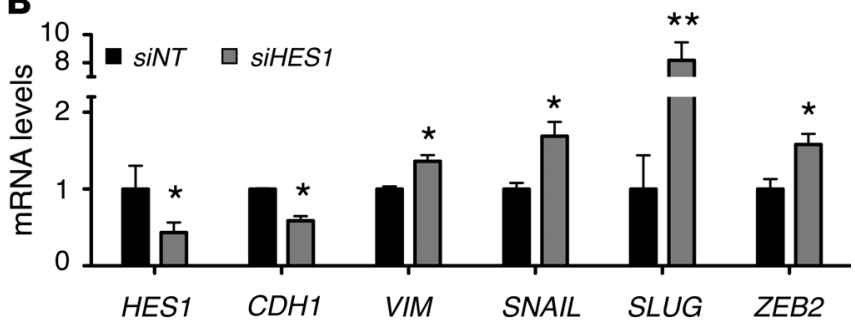

C

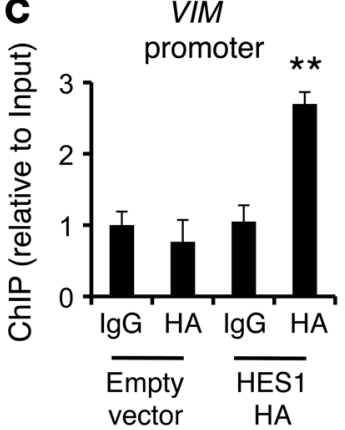

D

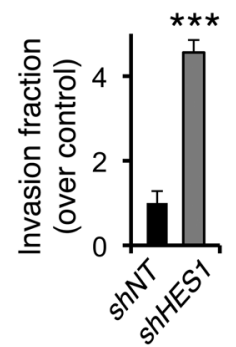

Invasion

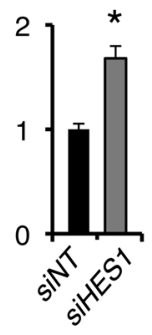

Figure 5. NOTCH inactivation promotes EMT in a cell-autonomous manner. Human urothelial carcinoma T24 cells were (A) treated with the $\gamma$-secretase inhibitor DBZ for 72 hours or (B) transfected with a nontargeting scrambled siRNA (siNT) or with siHES1 for 48 hours, and the mRNA from the indicated genes was assayed by qRT-PCR. (C) ChIP using the indicated antibodies was performed on T24 cells infected with a HA-tagged version of HES1 or an empty vector and using PCR primers directed against the VIM promoter. (D) T24 cells infected with nontargeting control (shNT) or shHES1 or transfected with siNT or siHES1 were placed in invasion chambers, and invading cells were counted by confocal microscopy 20 hours later. For all panels, data correspond to the average of 3 independent experiments $(n=3)$. Error bars represent SEM. ${ }^{*} P<0.05$, ${ }^{* *} P<0.01,{ }^{* *} P<0.001$, Student's $t$ test. 

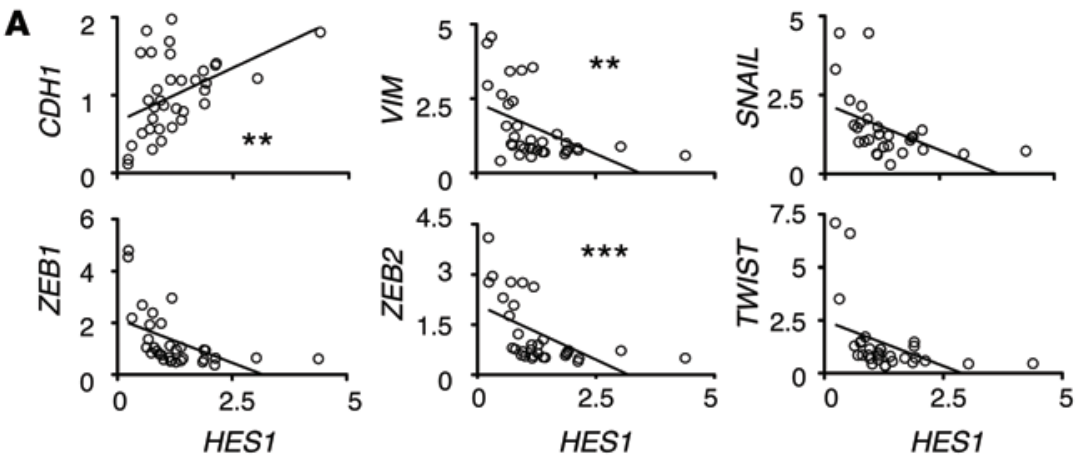

B

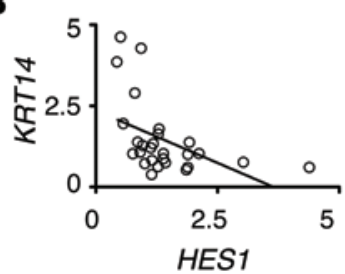

C

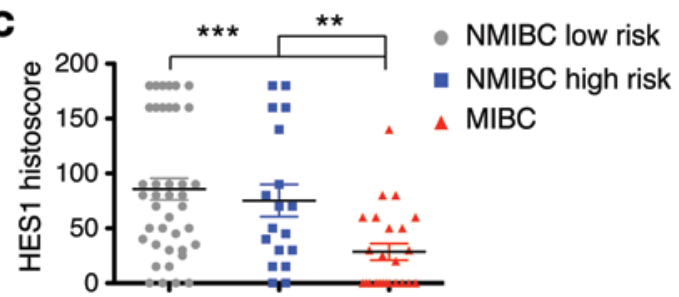

Figure 6. Low levels of HES1 correlate with EMT and invasiveness in human bladder cancers. (A) RNA samples from human bladder tumors were assayed for the indicated genes. The linear correlation of these genes with the corresponding expression of HES1 (the paired number of cases was between 30 and 35 ) is shown. ${ }^{* *} P<0.01,{ }^{* *} P<0.001$, Spearman test. (B) Correlation analysis of KRT14 and HES1 RNA expression in 27 paired cases. (C) Paraffin sections from human bladder tumor samples were graded into the indicated categories and stained for HES1 expression. The individual HES1 expression levels for each grade, nonmuscle invasive bladder cancer (NMIBC) low risk $(n=40)$, NMIBC high risk $(n=18)$, and muscle invasive bladder cancer (MIBC; $n=24$ ), are shown. ${ }^{* *} P<0.01$, ${ }^{* * *} P<0.001$, Mann-Whitney test. features, we treated the human bladder carcinoma cell line T24 with the $\gamma$-secretase inhibitor dibenzazepine (DBZ) (Figure 5A). DBZ treatment induced downregulation of HES1 and, concomitantly, an increase in VIM and a decrease in CDH1, thus supporting the notion that NOTCH inhibition induces EMT in human bladder cancer.

To directly test the impact of reduced HES1 levels on EMT, we transfected T24 cells with an siRNA against HES1 (siHES1, Figure 5B) or infected these cells with a lentivirus expressing an shRNA against HES1 (shHES1, Supplemental Figure 10) (of note, siHES1 and shHES1 target different sites of HES1 mRNA). Interestingly, both siHES1 and shHES1 induced a decrease in CDH1 expression and an increase in VIM expression (Figure 5B and Supplemental Figure 10), thus recapitulating our above observations in $\mathrm{NOTCH}$-deficient mice and human bladder carcinomas. EMT is a complex process promoted by several master regulators, including transcription factors from the Zeb, Twist and Snail families (30). Interestingly, in addition to VIM upregulation, HES1 downregulation also promoted an increase in SNAIL, SLUG, and ZEB2 expression when T24 cells were treated with DBZ (Figure 5A) or with siHES1 (Figure 5B).

HES1 is a well-known transcriptional repressor (26-28), and, based on this, we wondered whether it could bind to any of the EMT genes upregulated by HES1 inhibition (Figure 5, A and B). To test this, we performed a ChIP assay using a HA-tagged version of HES1 (31) and analyzed putative binding sites of HES1 in the proximal promoter regions (up to $5 \mathrm{~kb}$ upstream of the transcription initiation site) of VIM, SNAIL, SLUG, ZEB1, ZEB2, and TWIST. This approach is limited because relevant HES1 binding sites could be distant from the proximal promoter regions. In spite of this, we could detect a clear binding of HA-HES1 to the VIM promoter (Figure 5C) but not to the proximal promoters of the other tested genes (data not shown).

At a functional level, EMT is associated with an increase in cell invasion (30), and, therefore, we examined this cellular property in T24 cells treated with shHES1 or siHES1. For this, we performed Matrigel invasion chamber assays, and we observed increased invasion in shHES1- and siHES1-treated T24 cells compared with cells treated with a nontargeting control (Figure 5D).
We conclude that the NOTCH pathway, through HES1, contributes to the stabilization of the epithelial phenotype of bladder cancer cells.

Low HES1 expression correlates with aggressive cancer features. To further explore the possible association between HES1 and EMT, we examined two independent sets of human primary bladder cancer samples. One set consisted of RNA samples that were analyzed by cDNA microarrays and quantitative RT-PCR (qRT-PCR), and the other set consisted of paraffin blocks analyzed by immunohistochemistry. Using microarray-derived mRNA data (32), we observed a positive correlation between the levels of HES1 and $C D H 1$ and a remarkable inverse correlation between HES1 expression and EMT effectors, including VIM (Figure 6A). These observations were validated using qRT-PCR on a subset of the above RNA samples (Supplemental Figure 11). We also found an inverse correlation between HES1 and KRT14 mRNA levels (Figure 6B) and protein levels detected by immunohistochemistry (Supplemental Figure 12), which supports our previous analysis on the bladder carcinoma tumor set from TCGA (Supplemental Figure 9). Moreover, immunohistochemical analyses indicated that HES1 expression was significantly lower in the muscle invasive bladder carcinomas (MIBC) compared with that in nonmuscle invasive tumors (Figure 6C and Supplemental Figure 13). Finally, an independent analysis of Oncomine-deposited data (33-35) confirmed these clinical findings (Supplemental Figure 14). These observations indicate that low levels of HES1 associate with mesenchymal features and invasive properties in human bladder carcinomas.

\section{Discussion}

The NOTCH pathway can exert both oncogenic and tumorsuppressive effects. In general, NOTCH signaling is tumor suppressive in cancers of squamous histology, as is the case with esophageal, skin, and lung SCCs $(2,6-8,36)$. Since the urinary bladder is a stratified epithelium that can give rise to SCCs, we reasoned that the NOTCH pathway could be tumor suppressive in this tissue. Interestingly, although some observations 
have suggested that the NOTCH pathway could be oncogenic in bladder cancer $(37,38)$, there is substantial evidence indicating a tumor-suppressive role. In particular, a high fraction of bladder carcinomas present deletion of the long arm of chromosome 9q, where NOTCH1 is located (39), and low levels of NOTCH1 and JAGGED1 are associated with short survival in bladder cancer patients (40). Even more, a genome-wide association study in patients with bladder cancer found a significant association with a SNP in a region near to JAGGED1, and carriers of this SNP showed a trend to have lower JAGGED1 expression, again suggesting a tumor-suppressive role for the NOTCH pathway (41). Following on these observations, our analyses of NOTCH mutations from bladder cancer patients, mouse genetic models, cellbased assays, and human cancer samples provide solid evidence that the canonical NOTCH pathway plays a relevant tumor-suppressive role in bladder cancer. Importantly, as in other types of cancers, loss of function of the NOTCH pathway in the bladder is associated with SCCs.

Previous studies on mouse and human keratinocytes have proposed a mechanism for the tumor-suppressive activity of the NOTCH pathway in skin that relies in part on its role in terminal differentiation $(42,43)$. Specifically, in the absence of $\mathrm{NOTCH}$ signaling, keratinocytes do not fully differentiate and remain in a "stem-like" phenotype. In fact, in the esophagus, it has been demonstrated that lack of NOTCH signaling induces mutant clones that expand in a process known as field cancerization and that are at the origin of esophageal SCCs (44). A similar process could operate in the case of bladder cancer formation.

In our current study, we have found that inactivation of the NOTCH pathway induces EMT in squamous bladder cancer cells. Our mechanistic analyses imply that NOTCH signaling, through HES1, stabilizes epithelial features and prevents EMT in a cell-autonomous manner. We cannot exclude that other targets of the NOTCH pathway, like HEY1, could also contribute to the observed effects described here. In other cancer cellular contexts unrelated to stratified epithelia, such as prostate, breast, pancreas, lung adenocarcinoma, and others, the NOTCH pathway induces, rather than represses, EMT (45). Our data suggest that the repression of EMT could be a general tumor-suppressive mechanism of the NOTCH pathway in stratified epithelia. This, again, reflects the dual behavior of the NOTCH pathway, depending on the cellular type.

We and others have described antitumoral effects of pharmacological NOTCH inhibitors in preclinical models of T cell lymphoblastic leukemia, lung adenocarcinoma, pancreatic adenocarcinoma, and breast and intestinal tumors (46-50). Our data raise a note of caution on broad inhibition of the NOTCH pathway in the clinic, as this could concomitantly increase the incidence of tumors originated in stratified epithelia, such as bladder.

\section{Methods}

Further information can be found in the Supplemental Methods.

Animal experimentation. Mice were housed at the specific pathogen-free barrier area of the CNIO. Mice were observed on a daily basis and sacrificed when they showed overt signs of morbidity or tumors in accordance with the Guidelines for Humane Endpoints for Animals Used in Biomedical Research.
Microarray analysis. RNA was purified as described above and analyzed with an Agilent 2100 Bioanalyzer. Samples showing RNA integrity number above 8 were selected for microarray analysis. Genome-wide transcriptome experiments were performed using the Affymetrix HuGene-1_0-st-v1 microarray at the Genomics Facility of the Cancer Research Center (Salamanca, Spain) using standard procedures. Data sets have been deposited in GEO (accession no. GSE38264). The other two analyses described in Supplemental Figure 14 were performed using previously described available data sets (33-35).

Statistics. Unless otherwise specified, data are presented as mean \pm SEM. Two-tailed Student's $t$ test was carried out to assess the significance of expression levels in qRT-PCR, luciferase assay, and mouse IHC data. Correlation of protein or RNA expression data in human samples was evaluated using the Spearman correlation coefficient. Survival curves were tested by the log-rank test. The percentage of squamous tumors between groups and the clustering of human bladder tumors were evaluated using Fisher's exact test. HES1 protein levels in human bladder tumors were tested using the Mann-Whitney test. $P$ values lower than 0.05 were considered significant.

Study approval. All animal procedures were performed according to protocols approved by the CNIO-Instituto de Salud Carlos III (CNIO-ISCIII) Ethics Committee for Research and Animal Welfare. In the case of human samples, written informed consent was obtained from all patients, and the study was approved by the Ethical Committee for Clinical Research of each of the participating hospitals, namely, the University Hospital " 12 de Octubre" and the hospitals participating in the consortium EPICURO (51).

\section{Acknowledgments}

We thank E. Carrillo, D. Megías, M. Pérez, J. Soriano, N. Malats, M. Rava, M. Lozano, and A. del Rio for valuable contributions. A. Maraver is funded by the Miguel Servet Program of the Spanish Ministry of Health. P.J. Fernandez-Marcos is funded by the Asociación Española Contra el Cáncer (AECC). T.P. Cash and M. Martínez-Fernández are funded by the Juan de la Cierva Program from the Spanish Ministry of Economy and Competitiveness (MINECO). Work in the laboratory of M. Serrano is funded by CNIO and by grants from the European Research Council (advanced ERC grant), the Framework Program 7 of the European Union (RISK-IR), MINECO (SAF), the Regional Government of Madrid, the Botín Foundation, the Ramón Areces Foundation, and the AXA Foundation. Work in the laboratory of F.X. Real was supported, in part, by grants from the MINECO (Consolider ONCOBIO and SAF201115934-E), ISCIII (G03/174, 00/0745, PI051436, PI061614, G03/174, PI080440, PI120425, and Red Temática de Investigación Cooperativa en Cáncer), AECC (EU-FP7-201663), and NIH RO-1 (CA089715). Work in the CIEMAT was funded by MINECO (SAF2012-34378 and SAF2011-26122-C02-01), Comunidad Autónoma de Madrid (S2006/BIO-0232 and S2010/BMD-2470 [Oncocycle Programs]), ISCIII (RETIC RD06/0020/0029 and RD12/0036/0009) to J.M. Paramio, and MMA Foundation grant AP99782012 to M. Dueñas. We also acknowledge TCGA Research Network for freely providing genomic data. 
Address correspondence to: Antonio Maraver, Institut de Recherche en Cancérologie de Montpellier (Inserm U1194 - Université Montpellier), ICM Val d'Aurelle, 208 Rue des Apothicaires, F-34298 Montpellier Cedex 5, France. Phone:33467612395; E-mail: antonio.maraver@ inserm.fr. Or to: Manuel Serrano, Spanish National Cancer Research
Centre (CNIO), Melchor Fernández Almagro 3, Madrid 28029, Spain. Phone:34917328000-3430; E-mail:mserrano@cnio.es.

Antonio Maraver's present address is: Institut de Recherche en Cancérologie de Montpellier, Montpellier, France.
1. Guruharsha KG, Kankel MW, Artavanis-Tsakonas S. The Notch signalling system: recent insights into the complexity of a conserved pathway. Nat Rev Genet. 2012;13(9):654-666.

2. Ntziachristos P, Lim JS, Sage J, Aifantis I. From fly wings to targeted cancer therapies: a centennial for notch signaling. Cancer Cell. 2014;25(3):318-334.

3. Weng AP, et al. Activating mutations of NOTCH1 in human $\mathrm{T}$ cell acute lymphoblastic leukemia. Science. 2004;306(5694):269-271.

4. Puente XS, et al. Whole-genome sequencing identifies recurrent mutations in chronic lymphocytic leukaemia. Nature. 2011;475(7354):101-105.

5 . Westhoff B, et al. Alterations of the Notch pathway in lung cancer. Proc Natl Acad Sci U S A. 2009;106(52):22293-22298.

6. Agrawal N, et al. Exome sequencing of head and neck squamous cell carcinoma reveals inactivating mutations in NOTCH1. Science. 2011;333(6046):1154-1157.

7. Stransky N, et al. The mutational landscape of head and neck squamous cell carcinoma. Science. 2011;333(6046):1157-1160.

8. Wang NJ, et al. Loss-of-function mutations in Notch receptors in cutaneous and lung squamous cell carcinoma. Proc Natl Acad Sci U S A. 2011;108(43):17761-17766.

9. Klinakis A, et al. A novel tumour-suppressor function for the Notch pathway in myeloid leukaemia. Nature. 2011;473(7346):230-233.

10. Eble JN, Sauter G, Epstein JI, Sesterhenn IA. Pathology and Genetics of Tumours of the Urinary System and Male Genital Organs. Kleihues, P, Sobin, LH, eds. Lyon, France: IARCPress; 2004.

11. Scosyrev E, Yao J, Messing E. Urothelial carcinoma versus squamous cell carcinoma of bladder: is survival different with stage adjustment? Urology. 2009;73(4):822-827.

12. Cancer Genome Atlas Research Network. Comprehensive molecular characterization of urothelial bladder carcinoma. Nature. 2014;507(7492):315-322.

13. Balbas-Martinez C, et al. Recurrent inactivation of STAG2 in bladder cancer is not associated with aneuploidy. Nat Genet. 2013;45(12):1464-1469.

14. Guo G, et al. Whole-genome and whole-exome sequencing of bladder cancer identifies frequent alterations in genes involved in sister chromatid cohesion and segregation. Nat Genet. 2013;45(12):1459-1463.

15. Cordle J, et al. A conserved face of the Jagged/ Serrate DSL domain is involved in Notch trans-activation and cis-inhibition. Nat Struct Mol Biol. 2008;15(8):849-857.

16. Hass MR, Sato C, Kopan R, Zhao G. Presenilin: RIP and beyond. Semin Cell Dev Biol. 2009;20(2):201-210.

17. Kopan R, Ilagan MX. The canonical Notch signaling pathway: unfolding the activation mechanism. Cell. 2009;137(2):216-233.
18. Saura CA, et al. Loss of presenilin function causes impairments of memory and synaptic plasticity followed by age-dependent neurodegeneration. Neuron. 2004;42(1):23-36.

19. Tanigaki K, et al. Notch-RBP-J signaling is involved in cell fate determination of marginal zone B cells. Nat Immunol. 2002;3(5):443-450.

20. Puzio-Kuter AM, et al. Inactivation of p53 and Pten promotes invasive bladder cancer. Genes Dev. 2009;23(6):675-680.

21. Mao X, Fujiwara Y, Orkin SH. Improved reporter strain for monitoring Cre recombinase-mediated DNA excisions in mice. Proc Natl Acad Sci US A. 1999;96(9):5037-5042.

22. Shin $\mathrm{K}$, et al. Cellular origin of bladder neoplasia and tissue dynamics of its progression to invasive carcinoma. Nat Cell Biol. 2014;16(5):469-478.

23. Bryan GT. The pathogenesis of experimental bladder cancer. Cancer Res. 1977;37(8 pt 2):2813-2816.

24. Garcia-Cao I, et al. Tumour-suppression activity of the proapoptotic regulator Par4. EMBO Rep. 2005;6(6):577-583.

25. Chu PG, Lyda MH, Weiss LM. Cytokeratin 14 expression in epithelial neoplasms: a survey of 435 cases with emphasis on its value in differentiating squamous cell carcinomas from other epithelial tumours. Histopathology. 2001;39(1):9-16.

26. Espinosa L, et al. The Notch/Hes1 pathway sustains NF-kappaB activation through CYLD repression in T cell leukemia. Cancer Cell. 2010;18(3):268-281.

27. Maraver A, et al. Therapeutic effect of gamma-secretase inhibition in KrasG12V-driven non-small cell lung carcinoma by derepression of DUSP1 and inhibition of ERK. Cancer Cell. 2012;22(2):222-234.

28. Palomero T, et al. Mutational loss of PTEN induces resistance to NOTCH1 inhibition in T-cell leukemia. Nat Med. 2007;13(10):1203-1210.

29. Wendorff AA, et al. Hes1 is a critical but context-dependent mediator of canonical Notch signaling in lymphocyte development and transformation. Immunity. 2010;33(5):671-684.

30. Nieto MA. Epithelial plasticity: a common theme in embryonic and cancer cells. Science. 2013;342(6159):1234850.

31. Real PJ, et al. $\gamma$-Secretase inhibitors reverse glucocorticoid resistance in $\mathrm{T}$ cell acute lymphoblas tic leukemia. Nat Med. 2009;15(1):50-58.

32. Santos M, et al. In vivo disruption of an Rb-E2FEzh2 signaling loop causes bladder cancer. Cancer Res. 2014;74(22):6565-6577.

33. Rhodes DR, et al. ONCOMINE: a cancer microarray database and integrated data-mining platform. Neoplasia. 2004;6(1):1-6.

34. Sanchez-Carbayo M, Socci ND, Lozano J, Saint F, Cordon-Cardo C. Defining molecular profiles of poor outcome in patients with invasive bladder cancer using oligonucleotide microarrays. JClin Oncol. 2006;24(5):778-789.

35. Lee JS, et al. Expression signature of E2F1 and its associated genes predict superficial to invasive progression of bladder tumors. JClin Oncol. 2010;28(16):2660-2667.

36. Gao YB, et al. Genetic landscape of esophageal squamous cell carcinoma. Nat Genet. 2014;46(10):1097-1102.

37. Abdou AG, El-Wahed MM, Kandil MA, Samaka RM, Elkady N. Immunohistochemical analysis of the role and relationship between Notch-1 and Oct-4 expression in urinary bladder carcinoma. APMIS. 2013;121(10):982-996.

38. Li W, et al. High expression of Notch ligand Jagged 2 is associated with the metastasis and recurrence in urothelial carcinoma of bladder. Int JClin Exp Pathol. 2013;6(11):2430-2440.

39. Hirao S, et al. Loss of heterozygosity on chromosome $9 \mathrm{q}$ and $\mathrm{p} 53$ alterations in human bladder cancer. Cancer. 2005;104(9):1918-1923.

40. Shi TP, et al. Association of low expression of notch1 and jagged-1 in human papillary bladder cancer and shorter survival. JUrol. 2008;180(1):361-366.

41. Rafnar T, et al. Genome-wide association study yields variants at 20p12.2 that associate with urinary bladder cancer. Hum Mol Genet. 2014;23(20):5545-5557.

42. Nickoloff BJ, Qin JZ, Chaturvedi V, Denning MF, Bonish B, Miele L. Jagged-1 mediated activation of notch signaling induces complete maturation of human keratinocytes through NF- $\kappa \mathrm{B}$ and PPAR $\gamma$. Cell Death Differ. 2002;9(8):842-855.

43. Rangarajan A, et al. Notch signaling is a direct determinant of keratinocyte growth arrest and entry into differentiation. EMBO J. 2001;20(13):3427-3436.

44. Alcolea MP, Greulich P, Wabik A, Frede J, Simons $\mathrm{BD}$, Jones $\mathrm{PH}$. Differentiation imbalance in single oesophageal progenitor cells causes clonal immortalization and field change. Nat Cell Biol. 2014;16(6):615-622.

45. Espinoza I, Miele L. Deadly crosstalk: Notch signaling at the intersection of EMT and cancer stem cells. Cancer Lett. 2013;341(1):41-45.

46. Real PJ, Ferrando AA. NOTCH inhibition and glucocorticoid therapy in T-cell acute lymphoblastic leukemia. Leukemia. 2009;23(8):1374-1377.

47. Maraver A, Serrano M. Notching up a new therapeutic strategy for non-small cell lung carcinoma (NSCLC). Oncotarget. 2012;3(9):917-918.

48. Plentz R, et al. Inhibition of $\gamma$-secretase activity inhibits tumor progression in a mouse model of pancreatic ductal adenocarcinoma. Gastroenterology. 2009;136(5):1741-1749 e1746.

49. van Es JH, et al. Notch $/ \gamma$-secretase inhibition turns proliferative cells in intestinal crypts and adenomas into goblet cells. Nature. 2005;435(7044):959-963.

50. Schott AF, et al. Preclinical and clinical studies of $\gamma$ secretase inhibitors with docetaxel on human breast tumors. Clin Cancer Res. 2013;19(6):1512-1524.

51. Hernandez S, et al. Prospective study of FGFR3 mutations as a prognostic factor in nonmuscle invasive urothelial bladder carcinomas. J Clin Oncol. 2006;24(22):3664-3671. 